\title{
Pseudodifferential operators on ultrametric spaces and ultrametric wavelets
}

\author{
A.Yu.Khrennikov, S.V.Kozyrev
}

November 11, 2018

\begin{abstract}
A family of orthonormal bases, the ultrametric wavelet bases, is introduced in quadratically integrable complex valued functions spaces for a wide family of ultrametric spaces.

A general family of pseudodifferential operators, acting on complex valued functions on these ultrametric spaces is introduced. We show that these operators are diagonal in the introduced ultrametric wavelet bases, and compute the corresponding eigenvalues.

We introduce the ultrametric change of variable, which maps the ultrametric spaces under consideration onto positive half-line, and use this map to construct non-homogeneous generalizations of wavelet bases.
\end{abstract}

\section{Introduction}

The present paper is devoted to investigation of pseudodifferential operators on ultrametric spaces. Ultrametric pseudodifferential operators were considered in [1]10]. The simplest example among these operators is the Vladimirov $p$-adic fractional derivation operator, which can be diagonalized by the $p$-adic Fourier transform (the field of $p$-adic numbers is the example of ultrametric space). In the present paper we introduce a wide family of pseudodifferential operators on more general ultrametric spaces, which do not necessarily possess a group structure. Since there is no Fourier transform on general ultrametric space, the introduced pseudodifferential operators cannot be diagonalized using this method. Instead of this we introduce and apply the method of ultrametric wavelets.

In [11] the basis of $p$-adic wavelets in the space $L^{2}\left(Q_{p}\right)$ of quadratically integrable functions on the field of $p$-adic numbers was introduced and it was proven that this basis is a basis of eigenvectors for the Vladimirov operator. Also the relation to the standard wavelet analysis on real line was discussed. The wavelet analysis is a well established approach used in a broad field of applications (see for instance the review [12]). 
In paper [13] a family of pseudodifferential operators in the space $L^{2}\left(Q_{p}\right)$, diagonal in the basis of $p$-adic wavelets, but not diagonalizable by the Fourier transform, was built, and the corresponding eigenvalues were computed. In the present paper we generalize the results of papers [11] and [13] onto the case of a wide family of ultrametric spaces.

We introduce bases of ultrametric wavelets on the considered wide family of ultrametric spaces. These bases are analogous to $p$-adic wavelet basis, constructed in [11]. We prove that the ultrametric wavelet bases consist of eigenvectors for the introduced pseudodifferential operators. For this class of ultrametric spaces the wavelet analysis turns out to be an effective substitute for the Fourier analysis.

The consideration of the present paper is motivated by applications to ultrametric mathematical physics, for instance, to replica approach and disordered phenomena. For $p$-adic mathematical physics, see [1], 14]-[25], [1], where the models of mathematical physics are investigated with the help of $p$-adic analysis. For discussions of the replica approach and applications of ultrametricity in physics see for instance [26], 27]. In [21, 22] it was shown that the Parisi matrix describing the replica symmetry breaking (before the $n \rightarrow 0$ limit) is a discrete analogue of a $p$-adic pseudodifferential operator. In [23], 24] the Parisi matrices related to more general Abelian locally compact groups were considered. In 25] the relation of ultrametric diffusion and dynamics of macromolecules was discussed. Other applications of $p$ adic mathematical physics are mathematical models in biology and mental sciences [17, [18.

In the present paper we discuss the relation between the wavelet analysis and the ultrametric analysis. This discussion follows the line of research of [1], where a $p$-adic wavelet basis in the space of quadratically integrable functions with $p$ adic argument was introduced. To be more specific, in [1] it was shown that the natural map of $p$-adic numbers onto positive real numbers (called the $p$-adic change of variable) maps this basis onto the wavelet basis (in the space of quadratically integrable functions on positive real half-line) generated by the Haar wavelet (for $p=2$ ). For $p>2$ the $p$-adic change of variable maps the basis of $p$-adic wavelets onto the orthonormal basis in $L^{2}\left(\mathbf{R}_{+}\right)$which is a simple generalization of the basis generated by the Haar wavelet. The basis vectors are complex valued compactly supported stepwise functions, which take $p$ different values (the $p$-th complex roots of 1$)$.

In this paper we introduce specific maps for the considered ultrametric spaces onto positive real numbers. The introduced maps are surjective, they are one to one correspondences on the set of full measure, and are continuous. We call these maps the ultrametric changes of variable. They map ultrametric wavelet bases onto some new orthonormal bases in $L^{2}\left(\mathbf{R}_{+}\right)$. Note that these bases are analogous to the wavelet basis generated by the Haar wavelet. The main difference with the $p$-adic case is that the image of an ultrametric wavelet basis contains the vectors which are, up to shifts and dilations, the images of $p$-adic wavelets with different $p$. We call the image of ultrametric wavelet basis the non-homogeneous wavelet basis in $L^{2}\left(\mathbf{R}_{+}\right)$. The non-homogeneity here means that, unlike in the case of the usual wavelet bases, vectors of non-homogeneous wavelet basis can not be constructed using shifts and 
dilations of fixed wavelet.

The structure of the present paper is as follows.

In Section 2 we construct a family of the ultrametric spaces under consideration, and build measures on these spaces.

In Section 3 we introduce the orthonormal ultrametric wavelet bases in the spaces of quadratically integrable functions on spaces, considered in Section 2.

In Section 4 we introduce pseudodifferential operators, acting on complex valued functions on spaces, defined in Section 2, and prove that these pseudodifferential operators are diagonal in the bases of ultrametric wavelets.

In Section 5 we introduce the ultrametric change of variable, which maps the ultrametric spaces under consideration onto positive half-line, and use this map to construct non-homogeneous generalizations of wavelet bases.

\section{Construction of the ultrametric space}

In the present section we define a family of ultrametric spaces related to trees. A tree is a graph without loops. For general discussion of trees see [28].

An ultrametric space is a metric space with the metric $|x y|$ (the distance between $x$ and $y$ ), which satisfies the strong triangle inequality

$$
|a b| \leq \max (|a c|,|c d|), \quad \forall c
$$

Consider an arbitrary tree (finite or infinite), such that the path in the tree between arbitrary two vertices is finite, and the number of edges incident to each of the vertices is finite. If a vertex $I$ is incident to $p_{I}+1$ edges, we will say that the branching index of $I$ is $p_{I}$. Examples of this kind of trees are the Bruhat-Tits trees (when the branching index is constant).

The absolute of a tree will be an ultrametric space (with respect to the naturally defined metric). Consider two equivalent definitions of the absolute of the tree.

The first definition is as follows. The infinitely continued path with the beginning in vertex $I$ is a path with the beginning in $I$, which is not a subset of a larger path with the beginning in $I$. The space of infinitely continued paths in the tree, which begin in some vertex $R$ (that is, the root) is called the absolute of the tree. Obviously the definition of the absolute of the tree does not depend on the choice of $R$ (taking any other vertex $A$ leads to an equivalent definition).

The equivalent definition of the absolute is as follows: the absolute is the space of equivalence classes of infinitely continued paths in the tree, such that any two paths in one equivalence class coincide starting from some vertex (i.e. the tails of the paths in one equivalence class are the same). If we choose in each of the equivalence classes the paths, which begin in vertex $R$, we will reproduce the first definition.

We consider trees with a partial order (or directed trees), where the partial order is defined in the following way. Fix the vertex $R$ and the point $\infty$ at the absolute. To fix the point $\infty$ at the absolute means that have to fix the infinitely continued path $R \propto$ from the vertex $R$ to $\infty$. The point $\infty$ we will call the infinite point, or 
the infinity. We define the following natural partial order on the set of vertices of the tree: $J>I$ if $J$ belongs to the path $I \infty$.

We denote the absolute of the tree by $X$. Let us construct an ultrametric and a measure on $X$.

For the points $x, y$ of the absolute there exists a unique path $x y$ in the tree. The notation $x y$ should be understood in the following way. Since the points $x, y$ of the absolute are identified with the paths $R x$ and $R y$, the path $x y$ will be contained in $R x \cup R y$. Then there exists a unique vertex $A$ satisfying

$$
R x=R A x, \quad R y=R A y, \quad A x \bigcap A y=A
$$

The notation $A B C$ means that $A C=A B \cup B C$. Then

$$
x y=A x \bigcup A y
$$

Consider the paths $x \infty$ and $y \infty$. There exists a unique smallest (in the introduced partial order) vertex $I$ such that

$$
x \infty=x I \infty, \quad y \infty=y I \infty
$$

We have

$$
x \infty=x I \bigcup I \infty, \quad y \infty=y I \bigcup I \infty, \quad x y=x I \bigcup I y
$$

We have three possibilities.

1) Let $I>R$. Then

$$
x y \bigcap R \infty=I
$$

Consider the (non-maximal) path $R I=I_{0} I_{1} \ldots I_{k}, R=I_{0}<I_{1}<\ldots<I_{k}=I$.

Define the distance between $x$ and $y$ as the following product of branching indices:

$$
|x y|=\prod_{j=1}^{k} p_{I_{j}}
$$

2) Let $I \leq R$. Then the vertex $R$ lies at the path $I \infty$. In this case we have the path $R I=I_{0} I_{1} \ldots I_{k}, R=I_{0}>I_{1}>\ldots>I_{k}=I$.

Define the distance between $x$ and $y$ as follows:

$$
|x y|=\prod_{j=0}^{k-1} p_{I_{j}}^{-1}
$$

When $I=R$, the product above contains empty set of multipliers, and we define the distance as $|x y|=1$.

3) Let $I$ and $R$ are incomparable. In this case there exists a unique supremum $J$ in the sense of the introduced partial order in the tree:

$$
J=\sup (I, R)
$$

i.e. $J$ is the smallest vertex larger than both $R$ and $I$ :

$$
I \infty=I J \infty, \quad R \infty=R J \infty
$$


Consider the paths $R J=J_{0} J_{1} \ldots J_{k}, R=J_{0}<J_{1}<\ldots<J_{k}=J$; and $I J=$ $I_{0} I_{1} \ldots I_{l}, I=I_{0}<I_{1}<\ldots<I_{l}=J$ (correspondingly $J=J_{k}=I_{l}$ ).

Define the distance between $x$ and $y$ as follows:

$$
|x y|=\prod_{m=1}^{k} p_{J_{m}} \prod_{n=1}^{l} p_{I_{n}}^{-1}
$$

Summing up the above three cases, the introduced distance between $x$ and $y$ can be described as follows.

Put into correspondence to an edge in the tree the branching index of the largest vertex of the edge (this definition is correct, since any two vertices, connected by edge, are comparable).

For the points $x$ and $y$ of the absolute consider vertex $I$, where the paths $x \infty$ and $y \infty$ merge. Then the distance $|x y|$ is introduced as the product of branching indices of edges in the directed path $R I$ in the degrees \pm 1 , where branching indices of increasing edges are taken in the degree +1 , and branching indices of decreasing edges are taken in the degree -1 . Here an edge is called increasing, if the end of the edge is larger than the beginning, and is called decreasing in the opposite case.

The following lemma can be proved by direct computation.

Lemma 1 The function $|x y|$ is an ultrametric (i.e. it is nonnegative, equal to zero only for $x=y$, symmetric, and satisfies the strong triangle inequality):

$$
|x y| \leq \max (|x z|,|y z|), \quad \forall z
$$

Proof To prove that $|x y|$ is an ultrametric, it is sufficient to prove that $|x y|$ satisfies the strong triangle inequality (the other conditions, which are necessary for ultrametricity are obvious).

Consider the points $x, y, z$ at the absolute and the corresponding paths $x \infty, y \infty$, $z \infty$. Then the paths $x \infty, y \infty$ coincide, starting from some vertex $I$ (we consider these paths as increasing paths to the point $\infty$ ). Analogously, the paths $y \infty, z \infty$ coincide, starting from some vertex $J$; the paths $x \infty, z \infty$ coincide, starting from some vertex $K$.

Since vertices $I$ and $K$ lie at the increasing path $x \propto$, these vertices are comparable. Analogously, the vertices $I$ and $J$ are comparable; as well as the vertices $J$ and $K$. Therefore the set of vertices $I, J, K$ is an ordered set.

There are two possibilities: or $I=J=K$, or some of the vertices do not coincide. If $I=J=K$, then by (31), (41), (51)

$$
|x y|=|x z|=|y z|
$$

Let $I>J$. Then the increasing paths $y \infty$ and $z \infty$ coincide, starting from $J$, and coincide with the path $x \infty$, starting from $I$, which implies that $I=K$. Therefore, by (3), (4), (15)

$$
|x y|=|x z|>|y z|
$$


i.e. the strong triangle inequality is satisfied.

Analogously, with the other choice of the order on the set $I, J, K$ we again will obtain the strong triangle inequality, which finishes the proof of the lemma.

We have defined the ultrametric on the absolute of the tree. In the topology corresponding to the defined ultrametric, the absolute $X$ will be locally compact. For the Bruhat-Tits tree the construction of ultrametric reduces exactly to the definition of $p$-adic distance.

Define the measure $\mu$ on the absolute of the tree, which for the case of the Bruhat-Tits tree will reduce to the Haar measure on $p$-adic numbers. To define the measure $\mu$, it is enough to define this measure on the disks $D_{I}$, where $D_{I}$ is the set of all the infinitely continued paths incident to the vertex $I$ which intersect the path $I \infty$ only at the vertex $I$.

Define the diameter $d_{I}$ of the disk as the supremum of the distance $|x y|$ between the paths $I x$ and $I y$ in $D_{I}$. Then $D_{I}$ is the ball of radius $d_{I}$ with its center on any of $I x \in D_{I}$.

Definition 2 The measure $\mu\left(D_{I}\right)$ of the disk $D_{I}$ is equal to the disk diameter.

Since the disk $D_{I}$ contains $p_{I}$ maximal subdisks, which by definitions of the ultrametric and the measure have the measure $p_{I}^{-1} \mu\left(D_{I}\right)$, the measure $\mu$ is additive on disks. By additivity we can extend the measure on algebra generated by disks ( $\sigma$-additivity of the measure will follow from the local compactness of the absolute, analogously to the case of the Lebesgue measure). We denote $L^{2}(\mu, X)$ the space of the square integrable (with respect to the defined measure) functions on the absolute. Since the absolute $X$ is not a group, there is no Fourier transform in $L^{2}(\mu, X)$. We are nevertheless able to define the wavelet transform.

Define the enumeration on the set of directed edges (the edge is directed, or has a direction, if we distinguish the beginning and end of the edge). For each vertex $I$ in the tree we have $p_{I}+1$ edges incident to the vertex, $0 \leq p_{I}<\infty$. By definition there exists a unique edge incident to the path $I \infty$. Enumerate this edge by -1 , and enumerate all the other $p_{I}$ edges by $x_{I}=0, \ldots, p_{I}-1$ in an arbitrary way. Note that the direction of the edge is important: since every edge has a beginning and an end, it corresponds to two directed edges (with the opposite direction) with two different numerations. We also take all the edges at the path $R \propto$, directed from the $\infty$ to $R$ be enumerated by 0 (and by -1 if the edges are directed in the opposite way).

Define the following enumeration of the points of the absolute $X$ by sequences of indices. Consider the point $x$ of the absolute. Consider the paths $R x$ and $R \infty$. There exists a unique vertex $I$ such that

$$
R x=R I x, \quad R \infty=R I \infty, \quad x \infty=x I \bigcup I \infty
$$

It is obvious that $R$ and $I$ are comparable, i.e. we have two possibilities: $I \leq R$ or $I>R$. 
1) Let $I>R$ and the distance in the tree between $I$ and $R$ (the number of edges in the path $I R)$ is $\gamma$. Then we enumerate the vertices in the corresponding path $I x=I_{-\gamma} I_{-\gamma+1} \ldots$. The sequence corresponding to $x$ can be written:

$$
x=x_{I_{-\gamma}} x_{I_{-\gamma+1}} \ldots x_{I_{-1}}, x_{I_{0}} x_{I_{1}} \ldots
$$

Here $x_{J}$ are the numbers of the edges directed from the higher to the lower vertex in the path (this means that there is no -1 indices here, all the indices are in the set $\left.0, \ldots, p_{J}-1\right)$.

2) Let $I=R$. Then we enumerate the vertices in the path $R x=I_{0} I_{1} \ldots$ The sequence corresponding to $x$ can be written:

$$
x=0, x_{I_{0}} x_{I_{1}} \ldots
$$

This enumeration is the analogue of the expansion of a $p$-adic number into a series over the degrees of $p$ or of the expansion of a real number into infinite decimal fraction. In both these expansions, numbers (real or $p$-adic) are parameterized by sequences of digits. This suggests to call the introduced parameterization of the absolute the digital parameterization.

Remark The defined above parameterization allows to put in correspondence to the vertex $I$ the point of the absolute with the enumeration $I 0 \ldots$, which we will denote by the same symbol $I$.

\section{The wavelet basis in $L^{2}(\mu, X)$}

For the vertex $I$ of the tree, define the function $\Omega_{I}(x)$ on the absolute, which is equal to the characteristic function of the disk $D_{I}$.

Define the ultrametric wavelet as the function $\psi_{I j}(x)$ on the absolute, where $I$ is the vertex of the tree and $j=1, \ldots, p_{I}-1$, given by the formula

$$
\psi_{I j}(x)=\frac{e^{2 \pi i j x_{I} p_{I}^{-1}} \Omega_{I}(x)}{\sqrt{\mu\left(D_{I}\right)}}
$$

The point $x$ of the absolute is a class of equivalence of infinitely continued paths. In this class there exists a path which begins in the vertex $I$. Then $\Omega_{I}(x)$ is equal to 0 or 1 depending on the direction of this path at $I: \Omega_{I}(x)$ is equal to 0 if the edge of the path $x$ at vertex $I$ is directed onto infinity, and $\Omega_{I}(x)$ is equal to 0 otherwise.

Note that the definition of the wavelet depends on the enumeration of the edges of the tree (but the supports of the wavelet do not depend on the enumeration).

Theorem $3\left\{\psi_{I j}\right\}$ is an orthonormal system of functions in $L^{2}(\mu, X)$. If all the infinitely continued directed paths in the tree are infinite, then $\left\{\psi_{I j}\right\}$ is a basis in $L^{2}(\mu, X)$. 
Proof Consider the scalar product

$$
\left\langle\psi_{I j}, \psi_{I^{\prime} j^{\prime}}\right\rangle=\frac{1}{\sqrt{\mu\left(D_{I}\right) \mu\left(D_{I^{\prime}}\right)}} \int e^{-2 \pi i j x_{I} p_{I}^{-1}} e^{2 \pi i j^{\prime} x_{I^{\prime}} p_{I^{\prime}}^{-1} \Omega_{I}(x) \Omega_{I^{\prime}}(x) d \mu(x)}
$$

The expression above can be non-zero only when $I \geq I^{\prime}$ or $I \leq I^{\prime}$. Without loss of generality we choose $I \leq I^{\prime}$. In this case

$$
\Omega_{I}(x) \Omega_{I^{\prime}}(x)=\Omega_{I}(x)
$$

Consider $I<I^{\prime}$. Then for the integral at the RHS of (7) we get

$$
\frac{1}{\sqrt{\mu\left(D_{I}\right) \mu\left(D_{I^{\prime}}\right)}} e^{2 \pi i j^{\prime} x_{I^{\prime}} p_{I^{\prime}}^{-1}} \int e^{-2 \pi i j x_{I} p_{I}^{-1}} \Omega_{I}(x) d \mu(x)=0
$$

since $x_{I^{\prime}}$ is a constant on $D_{I}$.

Therefore, the scalar product (7) can be non-zero only for $I=I^{\prime}$, then we obtain for (7)

$$
\left\langle\psi_{I j}, \psi_{I j^{\prime}}\right\rangle=\frac{1}{\mu\left(D_{I}\right)} \int e^{2 \pi i\left(j^{\prime}-j\right) x_{I} p_{I}^{-1}} \Omega_{I}(x) d \mu(x)=\delta_{j j^{\prime}}
$$

We get for (7)

$$
\left\langle\psi_{I j}, \psi_{I^{\prime} j^{\prime}}\right\rangle=\delta_{I I^{\prime}} \delta_{j j^{\prime}}
$$

which proves that vectors $\psi_{I j}$ are orthonormal.

To prove that if all the infinitely continued directed paths in the tree are infinite the set of vectors $\left\{\psi_{I j}\right\}$ is an orthonormal basis (i.e. it is total in $L^{2}(\mu, X)$ ), we use the Parsevale identity. Since the set of indicators (characteristic functions) of the disks $D_{I}$ is total in $L^{2}(\mu, X)$, proving that $\left\{\psi_{I j}\right\}$ is a total system requires only to check the Parsevale identity for the indicator $\Omega_{I}(x)$.

We have for the normed indicator the following scalar product:

$$
\frac{1}{\sqrt{\mu\left(D_{J}\right)}}\left\langle\Omega_{J}, \psi_{I j}\right\rangle=\frac{1}{\sqrt{\mu\left(D_{I}\right) \mu\left(D_{J}\right)}}\left\langle\Omega_{J}(x), e^{2 \pi i j x_{I} p_{I}^{-1}} \Omega_{I}(x)\right\rangle
$$

which is equal to

$$
\sqrt{\frac{\mu\left(D_{J}\right)}{\mu\left(D_{I}\right)}} e^{2 \pi i j x_{I} p_{I}^{-1}}
$$

for $J<I$, and to zero otherwise.

This implies the following identity:

$$
\sum_{I j}\left|\frac{1}{\sqrt{\mu\left(D_{J}\right)}}\left\langle\Omega_{J}, \psi_{I j}\right\rangle\right|^{2}=\mu\left(D_{J}\right) \sum_{I>J ; j} \frac{1}{\mu\left(D_{I}\right)}=\mu\left(D_{J}\right) \sum_{I>J} \frac{p_{I}-1}{\mu\left(D_{I}\right)}
$$

Consider the increasing sequence $J \infty, J=I_{0}<I_{1}<\ldots$ of vertices starting from $J$. We will consider both the cases when this sequence is finite or infinite (when the sequence is finite, we will denote the largest vertex in this sequence by $I_{f}$; this 
vertex can be identified with the infinite point $\infty$ of the absolute). Since $f$ is the length of the sequence $J \infty$, for the case when the sequence $J \infty$ is infinite, we will say that $f$ is infinite.

The following property is satisfied

$$
\mu\left(D_{I_{k}}\right)=\mu\left(D_{J}\right) \prod_{l=1}^{k} p_{I_{l}}
$$

This implies for (9) the following

$$
\mu\left(D_{J}\right) \sum_{I>J} \frac{p_{I}-1}{\mu\left(D_{I}\right)}=\sum_{k=1}^{f} \frac{p_{I_{k}}-1}{\prod_{l=1}^{k} p_{I_{l}}}=\sum_{k=1}^{f}\left[\left(\prod_{l=1}^{k-1} p_{I_{l}}\right)^{-1}-\left(\prod_{l=1}^{k} p_{I_{l}}\right)^{-1}\right]
$$

which is equal to

$$
1-\left(\prod_{l=1}^{f} p_{I_{l}}\right)^{-1}
$$

when $f$ is finite, and to

$$
\lim _{f \rightarrow \infty}\left[1-\left(\prod_{l=1}^{f} p_{I_{l}}\right)^{-1}\right]=1
$$

when $f$ is infinite.

It means that if all the infinitely continued directed paths in the tree are infinite the Parsevale identity is satisfied, and that $\left\{\psi_{I j}\right\}$ is an orthonormal basis in $L^{2}(\mu, X)$, thus proving the theorem.

We call this basis the basis of the ultrametric wavelets. For the $p$-adic case this basis reduces to the basis of $p$-adic wavelets introduced in [1].

\section{Pseudodifferential operators}

In the present section we construct a family of ultrametric pseudodifferential operators, which will be diagonal in the basis of ultrametric wavelets.

Consider the operator in $L^{2}(\mu, X)$

$$
T f(x)=\int T(x, y)(f(x)-f(y)) d \mu(y)
$$

Introduce some conditions on the kernel $T(x, y)$ of the operator (10).

Definition 4 We consider the class of kernels $T(x, y)$, which are nonnegative and depend (as a function of two variables $x$ and $y$ ) only on the highest (in the sense of the partial order in the tree) point $A(x, y)$ lying at the path between $x$ and $y$ in the tree. 
Lemma 5 The function $T(x, y)$ is symmetric, positive and locally constant with respect to $y$ for a fixed $x$ (outside any vicinity of $x$ ), and, for an arbitrary fixed $x$, the following condition is satisfied:

$$
T(x, y)=\text { const }, \quad \text { if } \quad|x y|=\text { const }
$$

Theorem 6 The function of the form

$$
T(x, y)=\sum_{I} T^{(I)} \delta_{|I|,|x y|} \Omega_{I}(x)
$$

where $T^{(I)} \geq 0$, satisfies the conditions of lemma 5, and an arbitrary function satisfying (11) can be represented in the form (12).

Here $|I|=\mu\left(D_{I}\right)$ is the diameter if the disk $D_{I}$, which consists of the paths incident to the vertex $I$ and directed in the opposite direction to the infinity (and the disk diameter is equal to the measure of the disk). Remind that the function $\Omega_{I}(x)$ is the characteristic function of the disk $D_{I}$.

Proof The positivity of $T(x, y)$ is obvious.

Let us prove the symmetricity of $T(x, y)$. We have

$$
T(x, y)-T(y, x)=\sum_{I} T^{(I)} \delta_{|I|,|x y|}\left(\Omega_{I}(x)-\Omega_{I}(y)\right)
$$

In order to prove that this expression is equal identically to zero, consider the case when $x$ is such that the following characteristic function is non-zero: $\Omega_{I}(x)=1$. This implies

$$
|x I| \leq|I|
$$

If $\delta_{|I|,|x y|} \neq 0$, then

$$
|x y|=|I|
$$

Formulas (14), (15) and ultrametricity of the absolute imply that $\Omega_{I}(y)=1$. Therefore, the corresponding terms in (13) cancel. This proves that the $T(x, y)$ given in (12) is symmetric. Let us prove now that it satisfies (11).

Fix $x \in D_{I}$ at the absolute. Then for $y$ lying at the sphere with the center $x$ and the radius $|I|$ we have

$$
\delta_{|I|,|x y|}=1
$$

Also $\Omega_{I}(x)$ is a constant on this sphere. Therefore $T(x, y)$ will be a constant on the considered sphere and (11) will be satisfied.

This proves that $T(x, y)$ satisfying the conditions of the present theorem will satisfy lemma 5 .

Vice versa, it is easy to see that the kernel (12) for $x, y$ lying in the disk with the center $I$ and the radius $|I|$, and satisfying $|x y|=|I|$, takes the value $T^{(I)}$.

Since all the space $x, y \in X \times X$ is the disjoint union of such a subsets, therefore, taking an arbitrary positive $T^{(I)}$ we are able to construct an arbitrary kernel satisfying (111). This finishes the proof of the theorem. 
Theorem 7 Let the kernel (11) satisfies the condition of convergence of all the integrals in (17) for any I. Then the operator (10) is a selfadjoint (and moreover, positive) operator in $L^{2}(\mu, X)$ with a dense domain and the wavelets $\psi_{I j}$ are eigenvectors for the operator (10):

$$
T \psi_{I j}(x)=\lambda_{I} \psi_{I j}(x)
$$

with the eigenvalues

$$
\lambda_{I}=\int_{|I y|>|I|} T(I, y) d \mu(y)+T(I, I 1) \mu\left(D_{I}\right)
$$

Here in the notations $|I y|, T(I, y), T(I, I 1)$, symbol $I$ is the point of the absolute, corresponding to vertex $I$ in the sense of the remark at the end of Section 2. Vertex $I 1$ is the maximal vertex, which is less than $I$ and has the numeration, obtained from the numeration of $I$ by adding of 1 (in $T(I, I 1)$ we mean the corresponding points of the absolute).

Proof To prove the present theorem we use Lemma 5 . Consider the wavelet $\psi_{I j}$. Then

$$
T \psi_{I j}(x)=\int T(x y)\left(\psi_{I j}(x)-\psi_{I j}(y)\right) d \mu(y)
$$

Consider the following cases.

1) Let $x$ lies outside $D_{I}$. Then Lemma 5 implies

$$
T \psi_{I j}(x)=-T(x, I) \int \psi_{I j}(y) d \mu(y)=0
$$

Note that by (111) $T(x, I)$ does not depend on the enumeration of the points of the absolute.

2) Let $x \in D_{I}$. Then again by Lemma 5

$$
\begin{aligned}
& T \psi_{I j}(x)=\left(\int_{|x y|>|I|}+\int_{|x y|=|I|}+\int_{|x y|<|I|}\right) T(x, y)\left(\psi_{I j}(x)-\psi_{I j}(y)\right) d \mu(y)= \\
& =\left(\int_{|x y|>|I|}+\int_{|x y|=|I|}\right) T(x, y)\left(\psi_{I j}(x)-\psi_{I j}(y)\right) d \mu(y)= \\
& =\psi_{I j}(x) \int_{|x y|>|I|} T(x, y) d \mu(y)+\int_{|x y|=|I|} T(x, y)\left(\psi_{I j}(x)-\psi_{I j}(y)\right) d \mu(y)= \\
& =\psi_{I j}(x) \int_{|I y|>|I|} T(I, y) d \mu(y)+T(I, I 1) \psi_{I j}(x) \mu\left(D_{I}\right) p_{I}^{-1} \sum_{l=1}^{p_{I}-1}\left(1-e^{2 \pi i j l}\right)
\end{aligned}
$$

The last equality follows from Lemma 5 and the local constance of $\psi_{I j}$.

Since for $j=1, \ldots, p-1 \bmod p$ we have

$$
\sum_{l=1}^{p-1}\left(1-e^{2 \pi i p^{-1} j l}\right)=p
$$


we obtain

$$
T \psi_{I j}(x)=\psi_{I j}(x)\left(\int_{|I y|>|I|} T(I, y) d \mu(y)+T(I, I 1) \mu\left(D_{I}\right)\right)
$$

which gives (17). Therefore the operator $T$ is well defined on the basis in $L^{2}(\mu, X)$. Moreover, the obtained eigenvalues are nonnegative. This finishes the proof of the theorem.

The next proposition gives a simple representation for the eigenvalues of the operator (10) with the kernel (12).

Proposition 8 Let the following series converge:

$$
\sum_{J>R} T^{(J)} \mu\left(D_{J}\right)<\infty
$$

Then the operator (10) corresponding to the kernel (12) is a selfadjoint (and moreover, positive) operator in $L^{2}(\mu, X)$, which is diagonal in the basis of ultrametric wavelets and has the following eigenvalues in this basis:

$$
\lambda_{I}=T^{(I)} \mu\left(D_{I}\right)+\sum_{J>I} T^{(J)} \mu\left(D_{J}\right)\left(1-p_{J}^{-1}\right)
$$

Note that condition of convergence of the series (18) is equivalent to convergence of the integrals (17).

Proof Substituting (12) into (17) we get

$$
\begin{gathered}
\lambda_{I}=\int_{|I y|>|I|} \sum_{J} T^{(J)} \delta_{|J|,|I y|} \Omega_{J}(I) d \mu(y)+\sum_{J} T^{(J)} \delta_{|J|,|I, I 1|} \Omega_{J}(I) \mu\left(D_{I}\right)= \\
=\sum_{J>I} T^{(J)} \mu\left(D_{J}\right)\left(1-p_{J}^{-1}\right)+T^{(I)} \mu\left(D_{I}\right)
\end{gathered}
$$

if the corresponding series converge.

Here we use the property

$$
\delta_{|J|,|I, I 1|} \Omega_{J}(I)=\delta_{I J}
$$

Since every two paths in the tree, which go to infinity, coincide starting from some vertex, condition (18) is equivalent to

$$
\sum_{J>I} T^{(J)} \mu\left(D_{J}\right)<\infty, \quad \forall I
$$

This finishes the proof of the proposition. 


\section{Relation to wavelets on real line}

In 11] the relation between the basis $\left\{\psi_{\gamma j n}\right\}$ of $p$-adic wavelets and the basis of wavelets in the space of quadratically integrable functions $L^{2}\left(\mathbf{R}_{+}\right)$on positive halfline was discussed. The basis $\left\{\psi_{\gamma j n}\right\}$ was called the basis of $p$-adic wavelets, since after the natural map of $p$-adic numbers onto positive real numbers (called the $p$-adic change of variable) this basis maps onto the wavelet basis (in the space of functions on positive real half-line) generated by the Haar wavelet (for $p=2$ ). For $p>2$ the $p$-adic change of variable maps the basis of $p$-adic wavelets onto the orthonormal basis in $L^{2}\left(\mathbf{R}_{+}\right)$, which is a simple generalization of the basis generated by the Haar wavelet: the vectors of this basis are complex valued compactly supported stepwise functions, which take $p$ different values equal to the $p$-th complex roots of 1 .

The wavelet basis in $L^{2}\left(\mathbf{R}_{+}\right)$is a basis given by shifts and dilations of the mother wavelet function, cf. [12. The simplest example of such a function is the Haar wavelet

$$
\Psi(x)=\chi_{\left[0, \frac{1}{2}\right]}(x)-\chi_{\left[\frac{1}{2}, 1\right]}(x)
$$

(i.e, the difference of two characteristic functions).

The wavelet basis in $L^{2}(\mathbf{R})$ (or basis of multiresolution wavelets) is the basis

$$
\Psi_{\gamma n}(x)=2^{-\frac{\gamma}{2}} \Psi\left(2^{-\gamma} x-n\right), \quad \gamma \in \mathbf{Z}, \quad n \in \mathbf{Z}
$$

The $p$-adic wavelets $\psi_{\gamma j n}(x)$, see [11], are defined in the way similar to the definition of $\psi_{I j}(x)$ in the present paper:

$$
\psi_{\gamma j n}(x)=p^{-\frac{\gamma}{2}} \chi\left(p^{\gamma-1} j x\right) \Omega\left(\left|p^{\gamma} x-n\right|_{p}\right) ; \quad \gamma \in \mathbf{Z}, n \in Q_{p} / Z_{p}, j=1, \ldots, p-1
$$

The following $p$-adic change of variable was considered:

$$
\begin{aligned}
\eta: Q_{p} & \rightarrow \mathbf{R}_{+} \\
\eta: \sum_{i=\gamma}^{\infty} a_{i} p^{i} & \mapsto \sum_{i=\gamma}^{\infty} a_{i} p^{-i-1}, \quad a_{i}=0, \ldots, p-1, \quad \gamma \in \mathbf{Z}
\end{aligned}
$$

which maps the wavelet basis on the basis of $p$-adic wavelets. The following theorem was proven:

Theorem. For $p=2$ the map $\eta$, defined by (22), maps the orthonormal basis of wavelets in $L^{2}\left(\mathbf{R}_{+}\right)$(generated from the Haar wavelet) onto the basis of eigenvectors of the Vladimirov operator ( $p$-adic wavelets):

$$
\eta^{*}: \Psi_{\gamma \rho(n)}(x) \mapsto(-1)^{n} \psi_{\gamma 1 n}(x)
$$

For general $p$ the $p$-adic change of variable, applied to the basis of $p$-adic wavelets, will generate a basis in $L^{2}\left(\mathbf{R}_{+}\right)$of vectors which, up to multiplication by numbers, have the form

$$
\Psi_{\gamma n}^{(p)}(x)=p^{-\frac{\gamma}{2}} \Psi^{(p)}\left(p^{-\gamma} x-n\right), \quad \gamma \in \mathbf{Z}, \quad n \in \mathbf{Z}_{+}
$$


where $\mathbf{Z}_{+}$is the set of positive integers and

$$
\Psi^{(p)}(x)=\sum_{l=0}^{p-1} e^{2 \pi i l p^{-1}} \chi_{\left[l p^{-1},(l+1) p^{-1}\right]}(x)
$$

This basis is a generalization of the basis of wavelets, generated by the Haar wavelet (and can be extended into a basis in $L^{2}\left(\mathbf{R}_{+}\right)$, if we take $n \in \mathbf{Z}_{+}$).

Constructed in the present paper basis $\left\{\psi_{I j}\right\}$ gives rise to a new basis in $L^{2}\left(\mathbf{R}_{+}\right)$, which is a generalization of the wavelet basis.

In the present paper we build a generalization of the map $\eta$, which we will call the ultrametric change of variable and denote by $\rho: X \rightarrow \mathbf{R}_{+}$. For the point $x$ at the absolute

$$
x=x_{I_{\gamma}} x_{I_{\gamma+1}} \ldots x_{I_{-1}}, x_{I_{0}} x_{I_{1}} \ldots ; \quad x_{I}=0, \ldots, p_{I}-1, \quad \gamma \in \mathbf{Z}
$$

the map $\rho$ looks as follows

$$
\rho: x \mapsto \sum_{k=\gamma}^{-1} x_{I_{k}} \prod_{l=k}^{-1} p_{I_{l}}+\sum_{k=0}^{\infty} x_{I_{k}} \prod_{l=0}^{k} p_{I_{l}}^{-1}
$$

for negative $\gamma$ and

$$
\rho: x \mapsto \sum_{k=\gamma}^{\infty} x_{I_{k}} \prod_{l=0}^{k} p_{I_{l}}^{-1}
$$

for positive $\gamma$.

This map is not a one-to-one map (but it is a one-to-one map almost everywhere). The map $\rho$ is continuous and moreover, one can prove the following lemma:

Lemma 9 The map $\rho$ satisfies the Hölder inequality

$$
|\rho(x)-\rho(y)| \leq|x y|
$$

Note that $|\cdot|$ at the LHS of (26) is the modulus of real argument and at the RHS is the ultrametric on the absolute $X$.

Proof Consider

$$
x=x_{I_{\alpha}} x_{I_{\alpha+1}} \ldots x_{I_{-1}}, x_{I_{0}} x_{I_{1}} \ldots ; \quad y=y_{J_{\beta}} y_{J_{\beta+1}} \ldots y_{J_{-1}}, y_{J_{0}} y_{J_{1}} \ldots
$$

where we assume without loss of generality that $\alpha \leq \beta$. For simplicity we assume $0 \leq \alpha \leq \beta$. In this case

$$
\begin{array}{ll}
x=0,0 \ldots 0 x_{I_{\alpha}} \ldots ; & \rho(x)=\sum_{k=\alpha}^{\infty} x_{I_{k}} \prod_{l=0}^{k} p_{I_{l}}^{-1} \\
y=0,0 \ldots 0 y_{I_{\beta}} \ldots ; & \rho(y)=\sum_{k=\beta}^{\infty} y_{J_{k}} \prod_{l=0}^{k} p_{J_{l}}^{-1}
\end{array}
$$


Then

$$
|x y|=\prod_{l=0}^{\alpha-1} p_{I_{l}}^{-1}
$$

We have

$$
\begin{gathered}
\rho(x)-\rho(y)=\sum_{k=\alpha}^{\beta-1} x_{I_{k}} \prod_{l=0}^{k} p_{I_{l}}^{-1}+\sum_{k=\beta}^{\infty}\left[x_{I_{k}} \prod_{l=0}^{k} p_{I_{l}}^{-1}-y_{J_{k}} \prod_{l=0}^{k} p_{J_{l}}^{-1}\right]= \\
=|x y|\left(\sum_{k=\alpha}^{\beta-1} x_{I_{k}} \prod_{l=\alpha}^{k} p_{I_{l}}^{-1}+\sum_{k=\beta}^{\infty}\left[x_{I_{k}} \prod_{l=\alpha}^{k} p_{I_{l}}^{-1}-y_{J_{k}} \prod_{l=\alpha}^{k} p_{J_{l}}^{-1}\right]\right) \leq \\
\leq|x y|\left(\sum_{k=\alpha}^{\beta-1}\left(p_{I_{k}}-1\right) \prod_{l=\alpha}^{k} p_{I_{l}}^{-1}+\sum_{k=\beta}^{\infty}\left(p_{I_{k}}-1\right) \prod_{l=\alpha}^{k} p_{I_{l}}^{-1}\right)= \\
=|x y| \sum_{k=\alpha}^{\infty}\left(p_{I_{k}}-1\right) \prod_{l=\alpha}^{k} p_{I_{l}}^{-1}=|x y| \lim _{f \rightarrow \infty}\left(1-\prod_{l=\alpha}^{f} p_{I_{l}}^{-1}\right)=|x y|
\end{gathered}
$$

which finishes the proof of the lemma.

Lemma 10 The map $\rho$ satisfies the conditions

$$
\begin{gathered}
\rho: D_{I} \rightarrow \rho(I)+\left[0, \mu\left(D_{I}\right)\right] \\
\rho: X \backslash D_{I} \rightarrow \mathbf{R}_{+} \backslash\left\{\rho(I)+\left[0, \mu\left(D_{I}\right)\right]\right\}
\end{gathered}
$$

up to a finite number of points.

Note that here we identify the vertex $I$ and the point at the absolute with the enumeration $I 0 \ldots$

Proof For the vertex $I$, consider the points

$$
I=x_{I_{\alpha}} x_{I_{\alpha+1}} \ldots x_{I_{-1}}, x_{I_{0}} x_{I_{1}} \ldots x_{I_{\beta-1}} 0 \ldots
$$

and

$$
\tilde{I}=x_{I_{\alpha}} x_{I_{\alpha+1}} \ldots x_{I_{-1}}, x_{I_{0}} x_{I_{1}} \ldots x_{I_{\beta-1}} p_{I_{\beta}}-1, \ldots
$$

The first is the point at the absolute $X$ corresponding to the vertex $I$, while the second is the first with the addition of the tail of $p_{I_{\beta}}-1, \ldots$

$$
\begin{gathered}
\rho(I)=\sum_{k=\alpha}^{-1} x_{I_{k}} \prod_{l=k}^{-1} p_{I_{l}}+\sum_{k=0}^{\beta-1} x_{I_{k}} \prod_{l=0}^{k} p_{I_{l}}^{-1} \\
\rho(\tilde{I})=\sum_{k=\alpha}^{-1} x_{I_{k}} \prod_{l=k}^{-1} p_{I_{l}}+\sum_{k=0}^{\beta-1} x_{I_{k}} \prod_{l=0}^{k} p_{I_{l}}^{-1}+\sum_{k=\beta}^{\infty}\left(p_{I_{k}}-1\right) \prod_{l=0}^{k} p_{I_{l}}^{-1}
\end{gathered}
$$


We have

$$
\begin{aligned}
\rho(\tilde{I})-\rho(I)= & \sum_{k=\beta}^{\infty}\left(p_{I_{k}}-1\right) \prod_{l=0}^{k} p_{I_{l}}^{-1}=\prod_{l=0}^{\beta-1} p_{I_{l}}^{-1} \sum_{k=\beta}^{\infty}\left(p_{I_{k}}-1\right) \prod_{l=\beta}^{k} p_{I_{l}}^{-1}= \\
& =\mu\left(D_{I}\right) \lim _{f \rightarrow \infty}\left(1-\prod_{l=\beta}^{f} p_{I_{l}}^{-1}\right)=\mu\left(D_{I}\right)
\end{aligned}
$$

Then

$$
\rho(\tilde{I})=\rho(I)+\mu\left(D_{I}\right)
$$

Using lemma 9, we obtain the proof of the lemma.

Lemma 11 The map $\rho$ maps the measure $\mu$ on the absolute onto the Lebesgue measure $l$ on $\mathbf{R}_{+}$: for any measurable subset $S \subset X$ we have:

$$
\mu(S)=l(\rho(S))
$$

or in symbolic notations

$$
\rho: d \mu(x) \mapsto d x
$$

Proof Lemma 10 implies that disks in $X$ map onto closed intervals in $\mathbf{R}_{+}$with conservation of measure. The map $\rho: X \rightarrow \mathbf{R}_{+}$is surjective, and since nonintersecting disks map onto intervals that do not intersect or have intersection of the measure zero (by lemma 10), this proves the lemma.

Therefore the corresponding conjugated map

$$
\begin{gathered}
\rho^{*}: L^{2}\left(\mathbf{R}_{+}\right) \rightarrow L^{2}(X) \\
\rho^{*} f(x)=f(\rho(x))
\end{gathered}
$$

is a unitary operator. The inverse to this map will map basis of ultrametric wavelets on the absolute $X$ on some basis of functions on real positive half-line.

This and Lemmas [10, 11] suggest the following definition:

Definition 12 We call the basis $\left\{\Psi_{I j}\right\}$ in $L^{2}\left(\mathbf{R}_{+}\right)$, where $\Psi_{I j}=\rho^{-1 *} \psi_{I j}$, the basis of nonhomogeneous wavelets on positive real half-line.

The map $\rho^{-1 *}$ between spaces of quadratically integrable functions is well defined since the map $\rho$ is one to one on the set of a complete measure.

We see that using the ultrametric change of variable $\rho$ we can define the new examples of wavelets in $L^{2}\left(\mathbf{R}_{+}\right)$. The name nonhomogeneous wavelets means that the basis $\rho^{-1 *}\left\{\psi_{I j}\right\}$ in $L^{2}\left(\mathbf{R}_{+}\right)$lacks translation invariance (the shift of the wavelet is not necessarily a wavelet, while for usual multiresolution wavelets this would be true).

The basis of nonhomogeneous wavelets combines wavelets corresponding to $p$ adic wavelets with different $p$. 


\section{Acknowledgements}

The authors would like to thank I.V.Volovich, G.Parisi and V.A.Avetisov for fruitful discussions and valuable comments. One of the authors (A.Kh.) would like to thank S.Albeverio for fruitful discussions and support of $p$-adic investigations. This paper has been partly supported by EU-Network "Quantum Probability and Applications". One of the authors (S.K.) has been partly supported by the Dynasty Foundation, CRDF (grant UM1-2421-KV-02), The Russian Foundation for Basic Research (project 02-01-01084), by the grant of the President of Russian Federation for the support of scientific schools NSh 1542.2003.1, and by the grant of The Swedish Royal Academy of Sciences on collaboration with scientists of former Soviet Union.

\section{References}

[1] Vladimirov V.S., Volovich I.V., Zelenov Ye.I. p-Adic Analysis and Mathematical Physics. Singapore: World Scientific, 1994.

[2] Vladimirov V.S. On the spectrum of some pseudodifferential operators on the field of $p$-adic numbers // Algebra and Analysis. 1990. V.2 N 6. p.107-124.

[3] Vladimirov V.S. On spectral properties of $p$-adic pseudodifferential operators of the Schrödinger type // Russ.Acad.Sci.Izv.Math. 1993. v.41. N1. p.55-73.

[4] Khrennikov A.Yu. Fundamental solutions over the field of $p$-adic numbers // Algebra and Analysis. 1992. v.4. N. 3. p.248-266.

[5] Albeverio S., Karwowosky $W$. A random walk on $p$-adic numbers, in "Stochastic Process-Physics and Geometry II " (S. Albeverio, U. Cattaneo, D. Merlini, Eds.), Proc. Locarno (1991), pp.61-74. Singapore: World Scientific, 1995.

[6] Kochubei A.N. Pseudo-Differential Equations and Stochastics over NonArchimedean Fields. New York: Marcel Dekker, 2001.

[7] Kochubei A.N. Fundamental solutions of pseudodifferential eqiations, related to $p$-adic quadratic forms // Izvestia Academii Nauk Seria Math. 1998. V. 62. N 6. P. 103-124.

[8] Ogielski A.T., Stein D.L. Dynamics on ultrametric spaces // Phys.Rev.Lett. 1985. V. 55. N 15. P. 1634-1637

[9] Schreckenberg $M$. Long run diffusion on ultrametric spaces // Zeitschrift fur Physik B - Condensed Matter. 1985. V. 60. P. 483-488

[10] Huberman B.A., Kerszberg M. Ultradiffusion: the relaxation on hierarchical systems // J.Phys.A: Math.Gen. 1985. V. 18. L331-L336

[11] Kozyrev S.V. Wavelet analysis as a $p$-adic spectral analysis // Russian Math. Izv. 2002. V. 66. N 2. P. 367. http://arxiv.org/abs/math-ph/0012019 
[12] Daubechies I. Ten Lectures on Wavelets, CBMS Lecture Notes Series, SIAM, Philadelphia, 1991.

[13] Kozyrev S.V. p-Adic pseudodifferential operators and $p$-adic wavelets // Theor. Math. Physics. 2004. V. 138. N 3, P. 322-332. http://arxiv.org/abs/math-ph/0303045

[14] Volovich I.V. p-Adic String // Class. Quantum Gravity. 1987. V. 4. L83-L87

[15] Aref'eva I.Ya., Dragovic B., Frampton P., Volovich I.V. Wave function of the universe and $p$-adic gravity // Mod. Phys. Lett. A. 1991. V. 6. p.4341-4358

[16] Khrennikov A.p-Adic valued distributions in mathematical physics. Dordrecht: Kluwer Academic Publ., 1994.

[17] Khrennikov A. Non-Archimedean Analysis: Quantum Paradoxes, Dynamical Systems and Biological Models. Dordrecht: Kluwer Academic Publishers, 1997.

[18] Khrennikov A. Classical and quantum mental models and Freud's theory of unconscious mind. Växjö University, Växjö, Sweden: Växjö University press, 2002.

[19] Khrennikov A. Yu., Non-Archimedean analysis and its applications. (In russian). Moscow: Nauka, Fizmatlit, 2003.

[20] Khrennikov A. p-adic discrete dynamical systems and their applications in physics and cognitive sciences // Russian J. Math. Phys. 2004. V. 11. N 1. p. $45-70$

[21] Avetisov V.A., Bikulov A.H., Kozyrev S.V. Application of $p$-adic analysis to models of spontaneous breaking of the replica symmetry // J. Phys. A: Math. Gen. 1999. V. 32. P. 8785-8791. http://arxiv.org/abs/cond-mat/9904360

[22] Parisi G., Sourlas N. p-Adic numbers and replica symmetry breaking // European Phys. J. B. 2000. V. 14. P. 535-542. http://arxiv.org/abs/cond-mat/9906095

[23] Carlucci, De Dominicis C. On the replica Fourier transform // Comptes Rendus Ac.Sci.Ser.IIB Mech.Phys. Chem.Astr. 1997. V. 325. P. 527. http://arxiv.org/abs/cond-mat/9709200

[24] De Dominicis C., Carlucci D.M., Temesvari T. Replica Fourier transform on ultrametric trees and block-diagonalizing multireplica matrices // J. Phys. I France. 1997. V. 7. P. 105-115. http://arxiv.org/abs/cond-mat/9703132

[25] Avetisov V.A., Bikulov A.H., Kozyrev S.V., Osipov V.A. p-Adic Models of Ultrametric Diffusion Constrained by Hierarchical Energy Landscapes // J. Phys. A: Math. Gen. 2002. V. 35. P. 177-189. http://arxiv.org/abs/cond-mat/0106506 
[26] Mezard M., Parisi G., Virasoro M. Spin-Glass Theory and Beyond. Singapore: World Scientific, 1987.

[27] Rammal R., Toulouse G., Virasoro M.A. Ultrametricity for physicists // Rev.Mod.Phys. 1986. V. 58. P. 765-788

[28] Serre J.P. Trees. New York, Berlin: Springer Verlag, 1980. 